CONFORMAL GEOMETRY AND DYNAMICS

An Electronic Journal of the American Mathematical Society

Volume 4, Pages 120-125 (December 15, 2000)

S $1088-4173(00) 00067-9$

\title{
A UNIQUENESS THEOREM FOR HARMONIC FUNCTIONS ON THE UPPER-HALF PLANE
}

\author{
BIAO OU
}

Abstract. Consider harmonic functions on the upper-half plane $R_{+}^{2}=$ $\{(x, y) \mid y>0\}$ satisfying the boundary condition $u_{y}=-\exp (u)$ and the constraint $\int_{R_{+}^{2}} \exp (2 u)<\infty$. We prove that all such functions are of form (1.2) below.

\section{INTRODUCTION}

Let $R_{+}^{2}$ be the upper-half plane $\{(x, y) \mid y>0\}$. Consider the problem

$$
\left\{\begin{array}{c}
\Delta u=0 \text { in } y>0, \\
u_{y}=-\exp (u) \text { on } y=0, \\
\int_{R_{+}^{2}} \exp (2 u)<\infty
\end{array}\right.
$$

Here we assume that $u(x, y)$ is a $C^{2}$ function on the upper-half plane down to the boundary. One easily verifies that functions of form

$$
u(x, y)=\ln \left(\frac{2 y_{1}}{\left(x-x_{1}\right)^{2}+\left(y+y_{1}\right)^{2}}\right),
$$

where $x_{1}$ is any real number and $y_{1}$ is any positive number, are solutions of (1.1). These solutions are in fact fundamental solutions of the Laplacian equation with a singularity at $\left(x_{1},-y_{1}\right)$ on the lower-half plane.

Problem (1.1) comes out of a geometric context. Suppose $u$ is a solution of (1.1). Then

$$
g_{i j}=\exp (2 u) \delta_{i j} \quad \text { for } i, j=1,2
$$

defines a Riemannian metric on the upper-half plane that is conformal to the Euclidean metric. In this metric, the Gaussian curvature is still zero everywhere (cf. [A] ); however, the geodesic curvature of the boundary $y=0$, with $x$ as parameter, is one at every point. The integral

$$
\int_{R_{+}^{2}} \exp (2 u)
$$

is the area of the upper-half plane. Essentially, we prove that the metric is in fact induced by a stereographic projection from the upper-half plane to a unit circle.

There is a counterpart of (1.1) in higher dimensions. There we consider positive harmonic functions on the upper-half space satisfying $u_{x_{n}}=-u^{n /(n-2)}(n \geq 3)$

Received by the editors August 14, 2000.

2000 Mathematics Subject Classification. Primary 53A30, 35J05, 30C15.

Key words and phrases. Harmonic, analytic, the Picard theorems.

(C)2000 American Mathematical Society 
on the boundary $x_{n}=0$. These functions also serve as the ones that attain a best constant in the Sobolev trace embedding theorem, (cf. [E]). The uniqueness theorem in higher dimensions has been established by Chipot et al [CSF], by Li and Zhu [LZ, and by the author [O] using different methods. The method used by the author in [O] does not extend to the plane. Fortunately, here we show that the classical complex analysis can be used to make up what was left out.

\section{An Analytic Function}

Let $z=x+i y$. We will identify $z$ with $(x, y)$. Let $u$ be a solution of (1.1) and let $v$ be a conjugate of $u$ satisfying the Cauchy-Riemann equations $u_{x}=v_{y}, \quad u_{y}=-v_{x}$. Define an analytic function by

$$
f(z)=\int_{0}^{z} \exp (u+i v) d z .
$$

We have

Lemma 2.1. The function $f(z)$ satisfies

(1) $\left|f^{\prime}(z)\right|=\exp (u(z)) \neq 0$ for all $z$ on the upper-half plane including the boundary;

(2) $f(z)$ maps the boundary $y=0$ into a unit circle and the curvature of the planar curve $\{f(x, 0) \mid-\infty<x<\infty\}$, with $x$ as parameter, is one for each $x ;$ and

(3) $\int_{R_{+}^{2}}\left|f^{\prime}(z)\right|^{2}=\int_{R_{+}^{2}} \exp (2 u)<\infty$.

Remark. The function $f(z)$ arises from the following geometric consideration. Let $I=\exp (2 u)\left(d x^{2}+d y^{2}\right)$ and $I I=0$. Then $I$ and $I I$ are the first and second fundamental forms of a parametric surface in $R^{3}$ because the Gauss-Codazzi equations are satisfied (cf. any textbook on differential geometry). In addition, by $I I=0$ the surface is planar. Thus $I$ and $I I$ define a conformal mapping from $R_{+}^{2}$ to a plane in $R^{3}$ and indeed $f(z)$ in $(2.1)$ is essentially such a mapping if we understand the complex plane as a subset of the three dimensional space.

Proof. We only need to verify the second statement by calculating the curvature of the planar curve $\{f(x, 0) \mid-\infty<x<\infty\}$ with $x$ as parameter. The curvature equals

$$
\begin{aligned}
& \frac{\operatorname{Im}\left(\overline{f^{\prime}(z)} f^{\prime \prime}(z)\right)}{\left|f^{\prime}(z)\right|^{3}} \quad(z=x=(x, 0)) \\
= & \operatorname{Im} \frac{\exp (u(z)-i v(z)) \exp (u(z)+i v(z))\left(u_{x}+i v_{x}\right)}{\exp (3 u(z))} \\
= & \operatorname{Im} \frac{1}{\exp (u(z))}\left(u_{x}+i v_{x}\right) \\
= & \frac{1}{\exp (u(z))}\left(v_{x}\right)=\frac{1}{\exp (u(z))}\left(-u_{y}\right)=1 .
\end{aligned}
$$

\section{The Uniqueness}

We will determine $u$ from the following uniqueness theorem on an analytic function $f$ satisfying the three conditions in Lemma 2.1. In the proof of Theorem 3.1 
below, we use the well-known Picard's Little Theorem and Picard's Great Theorem on several occasions. We refer to $[\mathrm{K}$ particularly for a proof of Picard's theorems using a geometric analysis technique.

Let $C$ be the unit circle that contains $\{f(x, 0) \mid-\infty<x<\infty\}$, let $D$ be the disk with $C$ as the boundary, and let $w_{0}$ be the center of $D$.

Theorem 3.1. The function $f(z)$ in Lemma 2.1 is of the form

$$
f(z)=w_{0}+e^{i \theta} \frac{z-z_{1}}{z-\overline{z_{1}}}
$$

where $\theta$ is a real number and $z_{1}=x_{1}+i y_{1}$ is a point on the upper-half plane.

Proof. Let $\Omega=f\left(R_{+}^{2}\right)$, the range set of $f$ on $R_{+}^{2}$. For every $w \in \Omega$, let $\chi(w)$ be the number of the $z$ in $R_{+}^{2}$ such that $f(z)=w$; that is, $\chi(w)$ is the number of times $f$ on $R_{+}^{2}$ takes on the value $w$. At the end we will see that $\Omega$ is nothing but $D$ and $\chi(w)$ is identically equal to one for every $w$ in $\Omega$. At present, however, it is only apparent that $\Omega$ is an open set because $f^{\prime}(z) \neq 0$ everywhere and thus $f$ is locally one-to-one. Next, we have that

$$
\int_{\Omega} \chi(w)=\int_{R_{+}^{2}}\left|f^{\prime}(z)\right|^{2} .
$$

The derivation of the above equality is as follows. Let $\chi_{R}(w)$ be the number of times $f$ on $R_{+}^{2} \cap B_{R}$ takes on the value $w$. Then $\chi_{R}(w)$ is piecewise constant and finite-valued. The equality

$$
\int_{\Omega} \chi_{R}(w)=\int_{R_{+}^{2} \cap B_{R}}\left|f^{\prime}(z)\right|^{2}
$$

is simply the result of a change of variables in the integration. Noting that $\chi_{R}(w) \rightarrow$ $\chi(w)$ from below and monotonically as $R \rightarrow \infty$, we have (3.2).

By $\int_{R_{+}^{2}}\left|f^{\prime}(z)\right|^{2}<\infty$ and (3.2), we know that $\chi(w)$ is finite almost everywhere on $\Omega$.

There are three possible cases and we examine them one by one: 1) $w_{0} \notin D$, 2) $w_{0} \in D$ and $\chi\left(w_{0}\right)$ is finite, and 3$) w_{0} \in D$ and $\chi\left(w_{0}\right)$ is infinite.

Case I. $w_{0}$, the center of $D$, is not in $\Omega$.

We prove that this case in fact cannot happen. Suppose there is such an $f$. Define the analytic extension

$$
g(z)= \begin{cases}f(z)-w_{0} & \text { if } z \in \overline{R_{+}^{2}}, \\ \frac{1}{\overline{f(\bar{z})}-\overline{w_{0}}} & \text { if } z \in R_{-}^{2} .\end{cases}
$$

Clearly, $g(z)$ is an entire function. Moreover, because $\chi(w)<\infty$ for almost every $w$ in $\Omega$ and thus $f(z)$ takes on almost every value finite times, $g(z)$ also takes on almost every value finite times. The Picard Little Theorem tells us that $g(z)$ must have a pole at infinity, for otherwise $g(z)$ takes on every value, with one possible exception, infinite times. That is, $g(z)$ must be a polynomial. But no polynomial maps the real line to a circle. We have come to the desired contradiction.

Case II. $w_{0} \in \Omega$ and $\chi\left(w_{0}\right)<\infty$.

Let $z_{1}, \ldots, z_{n}$ be all the $z$ in $R_{+}^{2}$ satisfying $f(z)=w_{0}$. Again define $g(z)$ as in (3.3). We note that this time $g(z)$ has simple roots at $z_{1}, \ldots, z_{n}$ and simple poles at 
$\overline{z_{1}}, \ldots, \overline{z_{n}}$. Again, $g(z)$ takes on almost every value finite times. The Picard Great Theorem tells us that $g(z)$ cannot have $\infty$ as an essential singular point. Thus $g(z)$ must be a rational function and it follows that

$$
g(z)=e^{i \theta} \frac{z-z_{1}}{z-\overline{z_{1}}} \cdots \frac{z-z_{n}}{z-\overline{z_{n}}}
$$

for a real number $\theta$. Furthermore, we show that $n$ must be one.

On the one side, we observe that $g^{\prime}(z)=f^{\prime}(z)$ on the upper-half plane and

$$
g^{\prime}(z)=-\overline{f^{\prime}(\bar{z})} /\left(\overline{f(\bar{z})}-\overline{w_{0}}\right)^{2}
$$

on the lower-half plane. Thus $g^{\prime}(z)$ has no roots and has only $\overline{z_{1}}, \ldots, \overline{z_{n}}$ as poles. On the other side, we show that $g^{\prime}(z)$ must have a root if $n \geq 2$. For this purpose, let $P_{1}(z)=\left(z-z_{1}\right) \ldots\left(z-z_{n}\right)=z^{n}+a_{n-1} z^{n-1}+\ldots+a_{0}$ and let $P_{2}(z)=\overline{P_{1}(\bar{z})}=$ $\left(z-\overline{z_{1}}\right) \ldots\left(z-\overline{z_{n}}\right)=z^{n}+\overline{a_{n-1}} z^{n-1}+\ldots+\overline{a_{0}}$. Then

$$
\begin{aligned}
g(z) & =e^{i \theta} \frac{z^{n}+a_{n-1} z^{n-1}+\cdots+a_{0}}{z^{n}+\overline{a_{n-1}} z^{n-1}+\cdots+\overline{a_{0}}} \\
& =e^{i \theta}\left(1+\frac{c_{m} z^{m}+\cdots+c_{0}}{z^{n}+\overline{a_{n-1}} z^{n-1}+\cdots+\overline{a_{0}}}\right),
\end{aligned}
$$

where $0 \leq m<n$ and $c_{m} \neq 0$. It's simple to have that

$$
g^{\prime}(z)=e^{i \theta} \frac{c_{m}(m-n) z^{m+n-1}+\cdots}{\left(z^{n}+\overline{a_{n-1}} z^{n-1}+\cdots+\overline{a_{0}}\right)^{2}}
$$

where the terms omitted in the numerator have powers less than $m+n-1$. Should $n$ be greater than or equal to two, $m+n-1 \geq 1$. Necessarily, the numerator would have at least a root. Furthermore, because this numerator also equals $P_{1}^{\prime}(z) P_{2}(z)-$ $P_{2}^{\prime}(z) P_{1}(z)$, which does not vanish at any of the $z_{1}, \ldots, z_{n}, \overline{z_{1}}, \ldots, \overline{z_{n}}$, we conclude that $g^{\prime}(z)$ would vanish at a point should $n \geq 2$. Therefore $n=1$ and

$$
g(z)=e^{i \theta} \frac{z-z_{1}}{z-\overline{z_{1}}}
$$

where $\theta$ is a real number and $z_{1}$ is a point on the upper-half plane.

Case III. $w_{0} \in \Omega$ and $\chi\left(w_{0}\right)=\infty$.

We show that this case also cannot happen. Let $w_{1}$ be another point inside $D$ other than the center $w_{0}$ and consider

$$
h(z)=w_{0}+\frac{\left(f(z)-w_{0}\right)-\left(w_{1}-w_{0}\right)}{1-\left(\overline{w_{1}-w_{0}}\right)\left(f(z)-w_{0}\right)} .
$$

Apparently, $h(z)$ also maps the real line into $C$. We choose $w_{1}$ so that 1$) f(z)$ takes on $w_{1}$ finite times, and 2) $f(z)$ takes on $w_{0}+1 /\left(\overline{w_{1}-w_{0}}\right)$ finite times-if $f(z)$ takes on $w_{0}+1 /\left(\overline{w_{1}-w_{0}}\right)$ at all. We choose $w_{1}$ this way to ensure that the function $h(z)-w_{0}$ have a finite number of simple zeros and poles. Such a $w_{1}$ always exists. We include here a few lines as a proof.

Consider $\chi(w)=0$ if $w \notin \Omega$. Then $\chi(w)<\infty$ for almost every $w$ on the whole complex plane. It follows that $\chi\left(w_{0}+1 /\left(\overline{w-w_{0}}\right)\right)<\infty$ for almost every $w$ on the whole complex plane as well. Hence

$$
\chi(w)+\chi\left(w_{0}+\frac{1}{\left(\overline{w-w_{0}}\right)}\right)<\infty
$$

almost everywhere. We can always choose a $w_{1}$ such that both $\chi\left(w_{1}\right)$ and $\chi\left(w_{0}+1 /\left(\overline{w_{1}-w_{0}}\right)\right)$ are finite. 
We proceed to define the analytic extension of $h(z)-w_{0}$ :

$$
g(z)= \begin{cases}h(z)-w_{0} & \text { if } z \in \overline{R_{+}^{2}}, \\ \frac{1}{\overline{h(\bar{z})}-\overline{w_{0}}} & \text { if } z \in R_{-}^{2} .\end{cases}
$$

The function $g(z)$ also has a finite number of simple zeros and poles on the whole complex plane. Again, $g(z)$ takes on almost every value finite times. The Picard Great Theorem says that $g(z)$ cannot have $\infty$ as an essential singular point. Thus $g(z)$ must be a rational function, but then $f(z)$ must be a rational function too, which led to $\chi\left(w_{0}\right)<\infty$, a contradiction to the assumption for the case.

In summary, we conclude that only Case II with $n=1$ may happen and from $(3.4)$,

$$
f(z)=w_{0}+e^{i \theta} \frac{z-z_{1}}{z-\overline{z_{1}}}
$$

It follows from Theorem 3.1 that a solution of (1.1) satisfies

$$
\begin{aligned}
u(x, y) & =u(z)=\ln \left|f^{\prime}(z)\right|=\ln \left|\frac{2 y_{1}}{\left(z-\overline{z_{1}}\right)^{2}}\right| \\
& =\ln \frac{2 y_{1}}{\left(x-x_{1}\right)^{2}+\left(y+y_{1}\right)^{2}},
\end{aligned}
$$

where $z_{1}=x_{1}+i y_{1}$ and $y_{1}>0$.

\section{A REMARK}

If we drop the restriction $\int_{R_{+}^{2}} \exp (2 u)<\infty$ in (1.1), there are infinitely many more solutions. In fact, let $h(z)$ be any entire analytic function such that $h(z)$ takes on real values for $z$ on the real line and $h^{\prime}(z) \neq 0$ for $z$ everywhere (for examples, $\exp (z), \exp (\exp (z)))$. Then $u(z)=\ln \left|f^{\prime}(z)\right|$ with

$$
f(z)=\frac{h(z)-i}{h(z)+i}
$$

is such a solution of (1.1). Particularly, the analytic function

$$
f(z)=\frac{e^{z}-i}{e^{z}+i}
$$

maps the real line to a half circle. The harmonic function $u(x, y)$ from this specific $f(z)$ is not of form (1.2) but satisfies the boundary condition $u_{y}=-\exp (u)$ and

$$
\int_{-\infty}^{\infty} \exp (u(x, 0)) d x=\int_{-\infty}^{\infty}\left|f^{\prime}(x, 0)\right| d x=\pi<\infty,
$$

and of course the integral $\int_{R_{+}^{2}} \exp (2 u)$ is infinite. This example also shows that the following assumption

$$
\int_{-\infty}^{\infty} \exp (u(x, 0)) d x<\infty
$$

cannot be used to replace the finiteness of the integral of $\exp (2 u)$ in (1.1). 


\section{ACKNOWLEDGMENT}

The author thanks his colleagues Professor H. Wente and Professor I. Sterling for valuable discussions on the geometric aspect of this work. He also thanks Professor Y.-Y. Li for having brought to his attention the problem addressed in the paper.

\section{REFERENCES}

[A] Aubin, T., Nonlinear analysis on manifolds, Monge-Ampere equations, Springer-Verlag, New York, 1982. MR 85j:58002

[ABR] Axler, S., Bourdon, P., and Ramey, W., Harmonic function theory, Springer-Verlag, New York, 1992. MR 93f:31001

[CY] Chang, A. and Yang, P., On uniqueness of solutions of nth order differential equations in conformal geometry, Math. Res. Lett. 4 (1997), 91 - 102. MR 97m:58204

[CL] Chen, W.-X. and Li, C.-M., Classification of solutions of some nonlinear elliptic equations, Duke Math. J. 63 (1991), 615 - 622. MR 93e:35009

[CSF] Chipot, M., Shafrir, I., and Fila, M., On the solutions to some elliptic equations with nonlinear Neumann boundary conditions, Adv. Differential Equations 1 (1996), 91 - 110.

[E] Escobar, J., Sharp constant in a Sobolev trace inequality, Indiana J. Math. 37 (1988), 687 - 698. MR 90a:46071

[K] Krantz, S., Complex analysis: the geometric viewpoint, Mathematical Association of America, Washington DC, 1990. MR 92a:30026

[LZ] Li, Y.-Y. and Zhu, M.-J., Uniqueness theorems through the method of moving spheres, Duke Math. J. 80 (1995), 383 - 417. MR 96k:35061

[O] Ou, B., Positive harmonic functions on the upper half space satisfying a nonlinear boundary condition, Differential Integral Equations 9 (5) (1996), 1157 - 1164. IMR 97i:35054

Department of Mathematics, University of Toledo, Toledo, Ohio 43606

E-mail address: bou@math.utoledo.edu 